\title{
Varicella Pneumonia in an Immunocompetent Adult
}

\author{
Thomas Sené $^{a}$ Sébastien Gallien ${ }^{a} \quad$ Constance Delaugerre ${ }^{b} \quad$ Anne-Marie Zagdanski ${ }^{c}$ \\ Jean-Michel Molina ${ }^{a}$ \\ Departments of ${ }^{a}$ Infectious and Tropical Diseases, ${ }^{b}$ Virology and ${ }^{c}$ Radiology, Saint-Louis Hospital, University of Paris \\ Diderot (Paris 7), Paris, France
}

All authors contributed equally to this work.

A 35-year-old heavy smoker presented with a 3-day history of fever, pruritic rash, cough and progressive dyspnea. His 3-year-old son had contracted chickenpox 15 days before admission. The father had no history of varicella or chickenpox vaccination.

Upon admission, his temperature was $38.7^{\circ} \mathrm{C}$, oxygen saturation $96 \%$ and respiratory rate $32 \mathrm{bpm}$. Physical examination revealed an exanthematous rash with lesions in different stages of development (vesicles, pustules and crusty lesions, see fig. 1) and bilateral basal subcrepitant rales. Blood tests were normal except for a plasma C-reactive protein level of $22 \mathrm{mg} / \mathrm{l}$. Chest radiography performed at admission showed bilateral interstitial nodular infiltrates. Varicella zoster virus DNA was detected by polymerase chain reaction in the serum.

The patient had no evidence of an underlying immunocompromised state: serological test for human immu- nodeficiency virus was negative, total lymphocyte count and levels of gammaglobulins were normal, and the patient did not report having had any noteworthy infections in the past several years.

Despite the initiation of intravenous acyclovir $(10 \mathrm{mg} /$ $\mathrm{kg} 3$ times daily) on the day of admission, his clinical state worsened with persistent high fever and severe dyspnea (respiratory rate: $40 \mathrm{bpm}$ and oxygen saturation 94\%, despite a flow rate of 6 liter/min). High-resolution computed tomography revealed small nodules scattered randomly throughout the lung parenchyma, patchy ground-glass opacities and basal alveolar consolidations (fig. 2). At day 4 , intravenous corticosteroids were started (methylprednisolone $1 \mathrm{mg} / \mathrm{kg}$ ) and progressively tapered down within a week, leading rapidly to apyrexia and normalization of all respiratory parameters within 4 days. The patient experienced a full recovery.

Fig. 1. Skin examination revealed a diffuse rash with vesicles, pustules and crusty lesions.

Fig. 2. High-resolution computed tomography revealed small nodules, equal or less than $5 \mathrm{~mm}$ in diameter (white triangle), scattered randomly throughout the lung parenchyma, patchy ground-glass opacities (white arrow) and basal alveolar consolidations (black star).
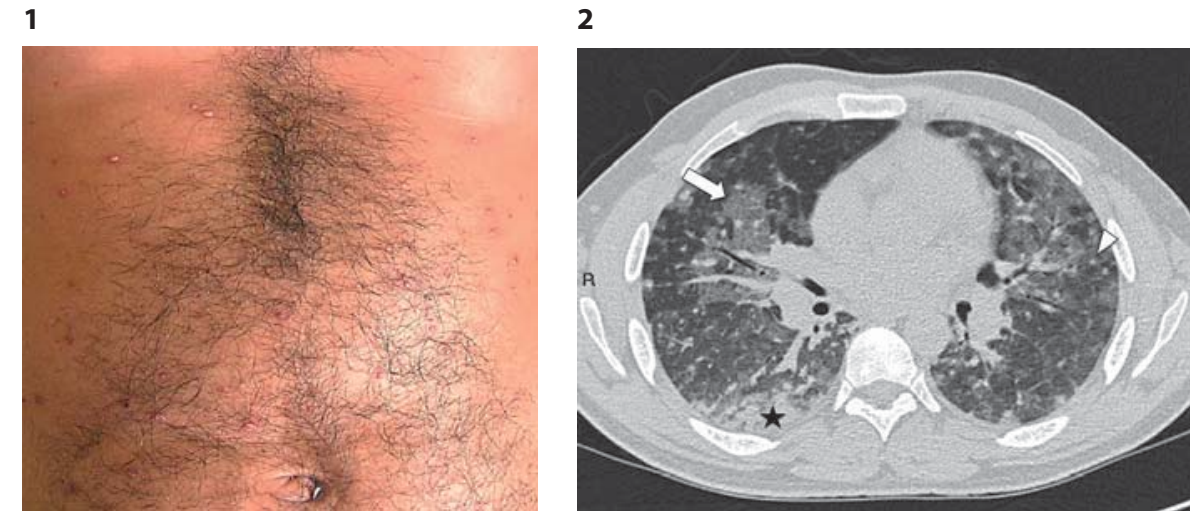